\title{
Comparative assessment of phototherapy protocols for reduction of oxidative stress in partially transected spinal cord slices undergoing secondary degeneration
}

\author{
Bethany Eve Ashworth ${ }^{1,2}$, Emma Stephens ${ }^{1,2}$, Carole A. Bartlett ${ }^{1}$, Stylianos Serghiou ${ }^{3}$, Marcus K. Giacci ${ }^{1}$, \\ Anna Williams ${ }^{3}$, Nathan S. Hart ${ }^{1,4}$ and Melinda Fitzgerald ${ }^{1 *}$
}

\begin{abstract}
Background: Red/near-infrared light therapy (R/NIR-LT) has been developed as a treatment for a range of conditions, including injury to the central nervous system (CNS). However, clinical trials have reported variable or sub-optimal outcomes, possibly because there are few optimized treatment protocols for the different target tissues. Moreover, the low absolute, and wavelength dependent, transmission of light by tissues overlying the target site make accurate dosing problematic.

Results: In order to optimize light therapy treatment parameters, we adapted a mouse spinal cord organotypic culture model to the rat, and characterized myelination and oxidative stress following a partial transection injury. The ex vivo model allows a more accurate assessment of the relative effect of different illumination wavelengths (adjusted for equal quantal intensity) on the target tissue. Using this model, we assessed oxidative stress following treatment with four different wavelengths of light: $450 \mathrm{~nm}$ (blue); $510 \mathrm{~nm}$ (green); $660 \mathrm{~nm}$ (red) or $860 \mathrm{~nm}$ (infrared) at three different intensities: $1.93 \times 10^{16}(\mathrm{low}) ; 3.85 \times 10^{16}$ (intermediate) and $7.70 \times 10^{16}$ (high) photons $/ \mathrm{cm}^{2} / \mathrm{s}$. We demonstrate that the most effective of the tested wavelengths to reduce immunoreactivity of the oxidative stress indicator 3-nitrotyrosine (3NT) was $660 \mathrm{~nm}$. $860 \mathrm{~nm}$ also provided beneficial effects at all tested intensities, significantly reducing oxidative stress levels relative to control ( $p \leq 0.05)$.

Conclusions: Our results indicate that R/NIR-LT is an effective antioxidant therapy, and indicate that effective wavelengths and ranges of intensities of treatment can be adapted for a variety of CNS injuries and conditions, depending upon the transmission properties of the tissue to be treated.
\end{abstract}

\section{Background}

Neurotrauma encompasses spinal cord injury (SCI) and traumatic brain injury (TBI) and can involve damage to both grey and white matter of the CNS [1]. Secondary degeneration is a hallmark of neurotrauma, with spreading damage contributing to deteriorating structure and function [2]. Oxidative stress contributes to secondary degeneration [3] and occurs when excess reactive oxygen

\footnotetext{
*Correspondence: lindy.fitzgerald@uwa.edu.au

${ }^{1}$ Experimental and Regenerative Neurosciences, School of Animal Biology, The University of Western Australia, Crawley, WA, Australia

Full list of author information is available at the end of the article
}

species (ROS) and/or reactive nitrogen species (RNS) overwhelm the endogenous antioxidant ability of the biological system [4]. While reactive species are essential for a range of normal cellular processes, excess leads to oxidative and nitrosative disruptions including protein nitration, DNA oxidation and lipid peroxidation [5-7], resulting in compromised oxidative metabolism, ATP depletion, necrosis, and dysregulated apoptosis [8]. If the excess production of reactive species can be limited, oxidative damage to DNA, lipids and proteins may be reduced and functional outcomes improved [9]. 
Red/near-infrared light therapy (R/NIR-LT), also known as photobiomodulation or phototherapy, is the therapeutic use of electromagnetic radiation at wavelengths characterized by relatively low energy densities in the red/near-infrared spectrum (600-1000 nm) [10]. R/NR-LT has been shown to have therapeutic effects in a range of CNS-specific injuries, resulting in improved functional recovery in cases of CNS injury [11], retinal degeneration $[12,13]$, stroke [14] and SCI [15] in rat models.

A proposed mechanism underlying R/NIR-LT therapy at the cellular level involves the activation of cytochrome c oxidase (COX) [14]. COX is complex IV of the mitochondrial respiratory chain and is considered to be one of the primary photoacceptors of visible and NIR light [1618]. When COX absorbs photons at specific wavelengths (600-1000 nm), it undergoes a conformational change, altering its redox state, increasing its activity resulting in increased levels of ATP [19], and improved mitochondrial function [14]. Irradiation with $670 \mathrm{~nm}$ light has been demonstrated to increase COX activity and reduce oxidative stress in an in vivo model of secondary degeneration [20]; these effects consistent with improvements in oxidative metabolism. Additional proposed mechanisms of action for R/NIR-LT include reduced inflammation and release of nitric oxide from COX [14, 21, 22].

Evidence to date indicates that R/NIR-LT is an effective and safe antioxidant therapy in a range of preclinical models as well as in clinical settings [20]. However, the NEST-3 clinical trial for stroke, delivering 630/830 nm light using transcranial laser therapy, failed an interim futility analysis [23], perhaps owing to the failure to implement an effective, optimized treatment protocol. It is difficult to identify effective treatment parameters for clinical trials of R/NIR-LT given the large range of wavelengths, intensities, pulse structure treatment durations, treatment intervals and delivery methods that have been employed to date in pre-clinical studies and which alter actual dose delivered [22, 24, 25]. In particular, varying degrees of penetrance of light, dependent on wavelength can confound attempts to optimise treatment parameters and dissect out potential mechanisms of positive effects. Pre-clinical in vivo data to date indicate that 670 and $810-830 \mathrm{~nm}$ light are effective wavelengths $[11,26]$. However, it is not yet clear whether efficacy is related to increased penetrance and thereby increased delivery of photons regardless of their delivery wavelength, or to specific effects of light at particular wavelengths. As such, light at short wavelengths may be even more effective than R/NIR wavelengths, if problems of penetration can be overcome.

Furthermore, effective parameters in one model do not necessarily apply to other models: a comparative study of
R/NIR-LT efficacy in four CNS injury models in rat (partial optic nerve transection, light-induced retinal degeneration, TBI and $\mathrm{SCI}$ ) indicated that it is necessary to optimize parameters individually for each type of CNS injury [26], perhaps due to differing penetrance of the light therapy. Therefore, to optimise treatment parameters of R/NIR-LT, a single, clinically relevant injury model where light can readily penetrate may assist.

Here, we adapt a well-established mouse organotypic spinal cord slice culture model which features the development of myelinated axons by 7 days in vitro (DIV) [27, 28] to rat, characterise the features of the culture system following partial transection injury and use it to determine the wavelengths and intensities of light therapy that can reduce an indicator of oxidative stress. A rat model was used in order to allow direct comparison to in vivo outcomes [26] in the same species. A model featuring three dimensional tissue architecture was desired as early attempts to optimise R/NIR-LT following excitotoxic insult to dissociated rat CNS cultures were unsuccessful [29]. Higher wavelengths of light are known to penetrate tissue more effectively due to the balance of increased absorption and reduced scattering $[16,30]$. However, if it is merely delivery of photons that provides beneficial effects, shorter wavelengths may be effective in the cell culture context where penetrance through overlying tissue is not required. We therefore assess a range of wavelengths from 450 to $860 \mathrm{~nm}$ at low, medium and high intensities, where the medium intensity for each wavelength delivers an equivalent number of photons as that delivered by the popular VET75 LED array (Quantum Devices).

\section{Results}

\section{Characterisation of injured spinal cord slice cultures from neonatal rat}

Spinal cord slices were prepared from postnatal day (P) 0-1 day old Piebald Virol Glaxo (PVG) rat pups. In P0-1 pups the uninjured spinal cord slice cultures contained cells that were myelin basic protein (MBP) immunopositive but had no myelinated axons at 4 DIV (Fig. 1A); some axons were myelinated by 7 DIV (Fig. 1B). Immunostained spinal cord sections collected from PVG rat pups similarly showed no myelinated axons in the uninjured spinal cord (Fig. 1C) or brain stem (Fig. 1D) at P0-1; or of P2 pups (not shown). Myelinated axons were, however, apparent in spinal cord sections at P5 (Fig. 1E), indicating that the development of myelination in neonatal rat in vitro parallels events in vivo. It is important to note that the myelination of axons in the uninjured rat spinal cord slice cultures was variable and that myelination was not always apparent. Electron microscopy revealed that myelin was spirally wrapped around some 


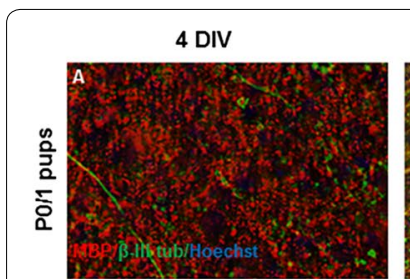

7 DIV: control

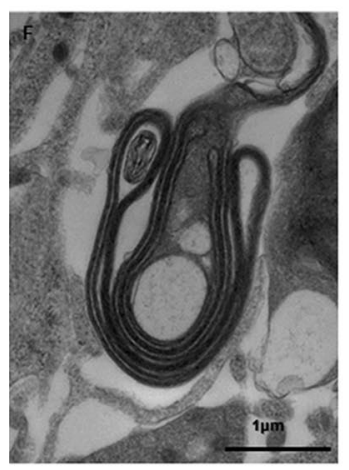

cut
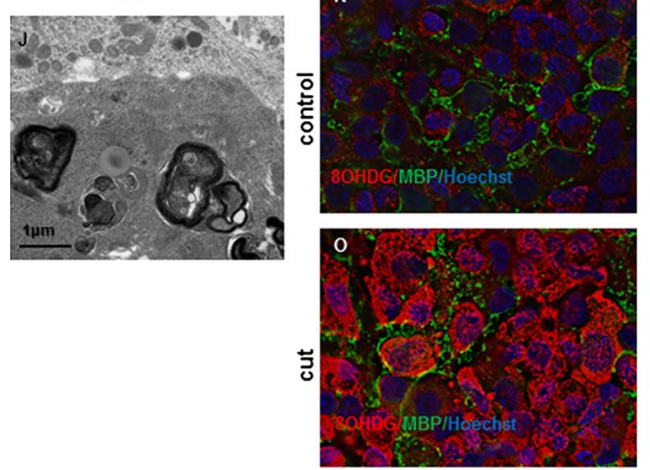
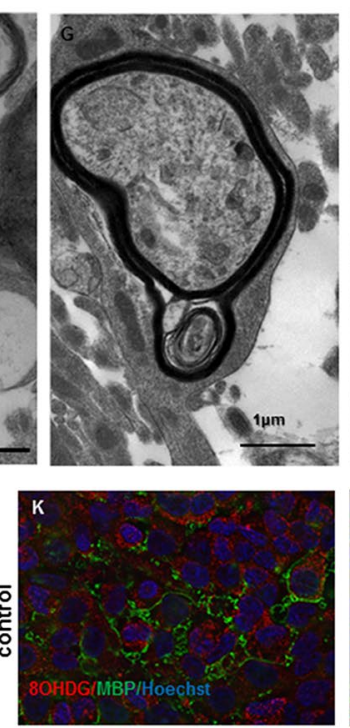

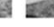
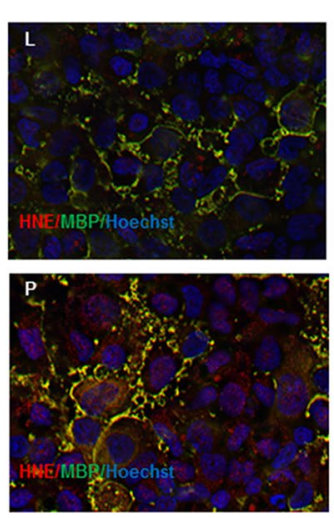

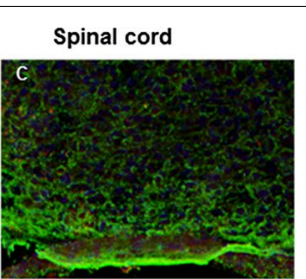

control
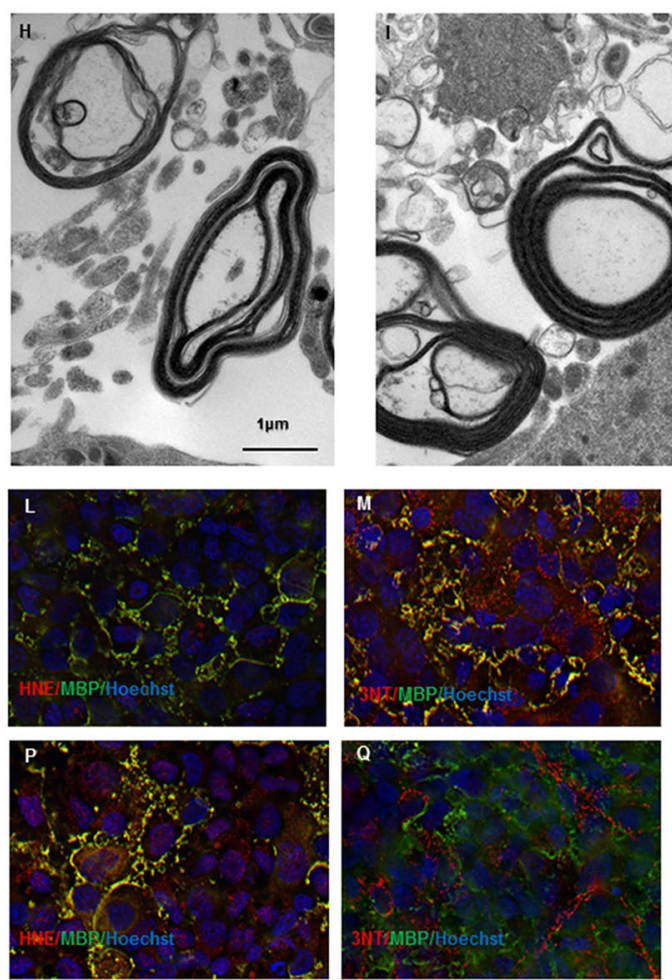

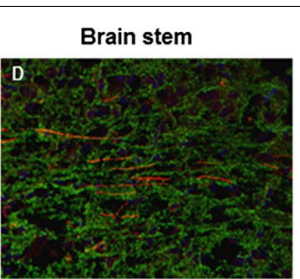

cut
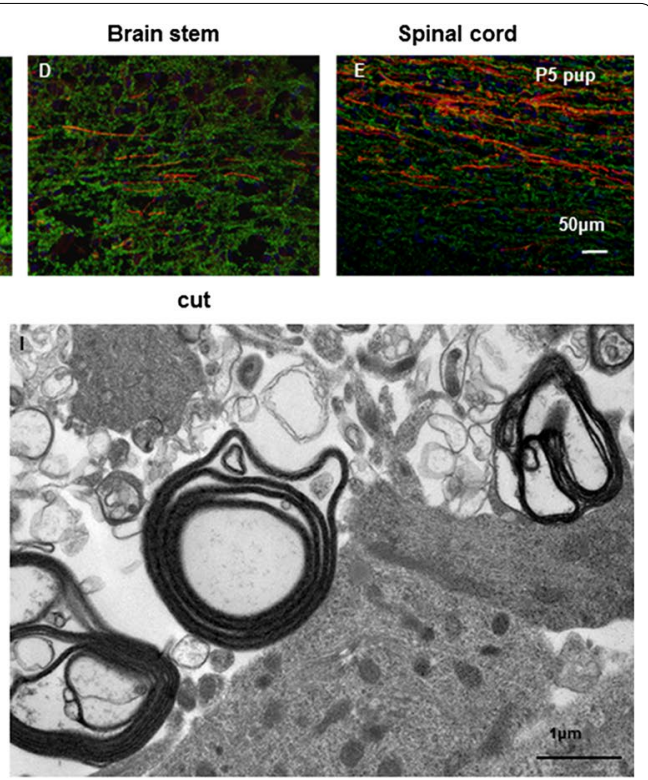

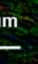
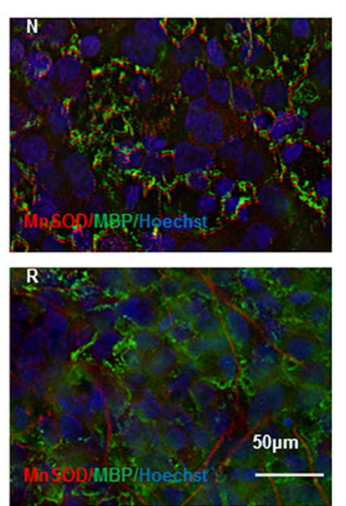

Fig. 1 Characterisation of spinal cord slice cultures from neonatal PVG rat. Myelinated axons, indicated by $\beta$-III tubulin (green) and MBP (red) colocalisation, were not apparent in spinal cord slice cultures from P0-1 rat pups at 4 DIV $(\mathbf{A})$ but were present at 7 DIV $(\mathbf{B})$, scale $=50 \mu \mathrm{m}$ for $\mathbf{A}$, B. Immunohistochemical assessment of perfusion fixed spinal cord (C) and brain stem (D) from P0-1 rat pups showed no evidence of myelinated axons; myelinated axons were observed in spinal cord from P5 pups (E), scale $=50 \mu \mathrm{m}$ for $\mathbf{C}-\mathbf{E}$. Electron microscopy of 7 DIV slice cultures revealed the presence of both relatively compact $(\mathbf{F}, \mathbf{G})$ and some decompacted myelin sheaths $(\mathbf{H})$ in uncut slice cultures; cut injury 24 h previously resulted in increasingly decompacted myelin (I) and aggregates of myelin debris (J), scale $=1 \mu \mathrm{m}$. Immunoreactivity of $80 \mathrm{HdG}(\mathbf{K})$ increased following cut injury $(\mathbf{O})$, as did immunoreactivity of $\operatorname{HNE}(\mathbf{L}, \mathbf{P})$, 3-NT $(\mathbf{M}, \mathbf{Q})$ and $\operatorname{MnSOD}(\mathbf{N}, \mathbf{R})$. Immunoreactivity of all oxidative stress indicators is shown in red, together with MBP immunoreactivity (green) and Hoechst nuclear stain (blue); representative images adjacent to the cut injury are shown. Note that multiple colour immunofluorescence was employed and as such, 8OHDG and HNE images show the same field of view, as do 3NT and MnSOD images, scale $=50 \mu \mathrm{m}$

axons, but compaction of myelin was not always complete (Fig. 1F-H). Myelination was most consistent in cultures derived from $\mathrm{P} 0-1$ rat pups and this age of pup was chosen for further analyses.

Spinal cord slice cultures from $\mathrm{P} 0-1$ rat pups were subjected to a cut injury after 7 DIV. Approximately half of the width of the slice was transected with a scalpel, resulting in transection of axons within the slice, as ascertained by subsequent immunohistochemical analyses. At 1 day following injury, myelinated axons were observed less frequently than in uninjured control slices; myelin was often decompacted (Fig. 1I) and aggregates of myelin debris were present (Fig. 1J). Cut injury resulted in increased immunoreactivity of a range of indicators of oxidative stress in the slice cultures $24 \mathrm{~h}$ following insult, both adjacent to the cut area and more distally (Fig. 1KR). 8-hydroxyguanosine (8OHDG) immunoreactivity, indicative of oxidative damage to DNA was increased in cell bodies, and as expected, was not co-localised with MBP (Fig. 1K, O); the area immediately adjacent to the cut injury is shown (Fig. 1O). Note that immunoreactivity of MBP was not clearly aligned along axons and in 
places was reminiscent of myelin debris (Fig. $1 \mathrm{~K}-\mathrm{R}$ ). As noted above, myelin debris was also observed by electron microscopy in cut injured cultures (Fig. 1I, J). 4-Hydroxynonenal (HNE) immunoreactivity, indicative of lipid peroxidation, was increased and co-localised with MBP immunoreactivity (Fig. 1L, P), as expected given the high lipid content of myelin. Increased immunoreactivity of 3 -nitrotyrosine (3NT), indicative of protein nitration by oxidative species, serves as a marker for oxidative/nitrosative stress in a range of cell types including myelinating oligodendrocytes [3]. Immunoreactivity of 3-NT was widespread in the tissue section and immunointensity was slightly increased following cut injury; staining was not apparent in MBP positive areas after injury (Fig. 1M, Q). Similarly, immunoreactivity of manganese superoxide dismutase (MnSOD), an antioxidant enzyme which converts superoxide to hydrogen peroxide, was slightly increased following injury and seldom co-localised with MBP immunoreactivity, particularly following cut injury (Fig. 1N, R).

Additional immunohistochemical analyses were conducted to ascertain whether 3NT colocalised with other cell types within the organotypic spinal cord slice cultures. 3NT immunoreactivity was broadly expressed throughout the spinal cord slice cultures (Fig. 2A, D), with some colocalisation with $\mathrm{NG}^{+} / \mathrm{Olig}^{+}$oligodendrocyte precursor cells (Fig. 2A-C, g arrows). 3NT occasionally colocalised with $\mathrm{IBA}^{+}$microglia and $\mathrm{ED} 1^{+}$ microglia/macrophages (Fig. 2D-F, h arrows); a control image where the primary antibody was omitted is provided (I). 3NT immunoreactivity was not different when comparing areas immediately adjacent to the cut injury, to areas more distal (Fig. 2J, K). The data indicate that the spread of oxidative/nitrosative stress occurred relatively rapidly, such that damage was uniformly distributed by $24 \mathrm{~h}$ after the injury. Given that the spinal cord slice cultures are approximately $4 \mathrm{~mm}$ in length, $3 \mathrm{NT}$ immunoreactivity spread more than $1.5 \mathrm{~mm}$ in $24 \mathrm{~h}$ of culture (L). While the size of spinal cord lesions in vivo is highly dependent upon the nature and severity of the injury, our data indicate the potential for significant spread of oxidative/nitrosative damage.

As a comparison, spinal cord slice cultures were prepared from P0-1 mouse pups, cultured for 10 DIV and then subjected to cut injury. Myelinated axons were clearly apparent in uninjured cultures (Fig. 3A, B). While lengths of MBP positive myelin were present 1 day after cut injury, myelin debris was apparent (Fig. 3E, F). By 7 days after the cut injury, some MBP immunoreactivity colocalised with neurofilament positive axons in the uncut section, but cut axons present in the lower half of the image were unmyelinated (Fig. 3I-L). MnSOD immunoreactivity was increased, particularly adjacent to the cut site (Fig. 3C, G), indicating oxidative stress that may be contributing to demyelination in the area with transected axons and reduced myelination. Despite some demyelination in the cut injured mouse slice cultures, it is apparent that myelination was more robust in the mouse slice cultures than in those from rat.

\section{Light treatment reduced an indicator of oxidative stress}

We used the partially transected rat in vitro spinal cord slice culture model system to compare effects of treatment with a range of wavelengths and intensities of light on oxidative stress, in tissue vulnerable to secondary degeneration. The slice cultures were injured at 7 DIV and tissue was assessed $24 \mathrm{~h}$ after cut injury (8DIV), immediately following two, 30 min light or control treatments $24 \mathrm{~h}$ apart. Indicators of oxidative stress examined were 3NT, HNE and 8OHDG. In $450 \mathrm{~nm}$ treated spinal cord slices we detected a significant decrease in the area of $3 \mathrm{NT}$ immunoreactivity above a set threshold at the intermediate and high intensities relative to control $(\mathrm{p}<0.05)$ (Fig. 4A, E-H), suggesting dose dependence at this wavelength. Immunoreactivity of $510 \mathrm{~nm}$ treated slices was significantly reduced at both low and high intensities of light compared to control $(\mathrm{p}<0.05)$ (Fig. 4B, E, I-K). In contrast, $660 \mathrm{~nm}$ light significantly reduced $3 \mathrm{NT}$ immunoreactivity consistently at all three tested intensities $(\mathrm{p} \leq 0.05)$ (Fig. $4 \mathrm{C}, \mathrm{E}, \mathrm{L}-\mathrm{N})$. Similarly, $860 \mathrm{~nm}$ light significantly reduced 3-NT immunoreactivity at all three intensities compared to control $(\mathrm{p} \leq 0.05)$ (Fig. 4D, E, O-Q), albeit to a lesser extent than $660 \mathrm{~nm}$ light and with a trend to a biphasic effect. Note that there were no statistically significant differences between the varying treatment intensities for $860 \mathrm{~nm}$, or $660 \mathrm{~nm}$ light ( $p>0.05)$. Specificity of the 3-NT antibody was demonstrated using established procedures [31], with increased 3-NT immunoreactivity in the cell culture supernatant of rat pheochromocytoma (PC12) neural like cells following treatment with SIN-1 (morpholinosydnonimine), a peroxynitrate generator [32], relative to untreated control (Fig. 5a). The effects of the full range of wavelengths and intensities on immunoreactivity of 8OHDG and HNE were assessed following cut injury in the slice cultures. There were no significant effects of any of the wavelengths and intensities of light on 8OHDG immunoreactivity, when considering both the area above a set threshold of intensity or the mean fluorescence intensity of the image (Fig. 5b, mean fluorescence intensity shown). Similar results were observed for HNE (not shown). Taken together our data indicate differential effects of R/ NIR-LT on oxidative damage, with beneficial effects on oxidative/nitrosative damage to protein, but not damage to DNA or lipid. A summary table of immunohistochemical outcomes of the study is provided in Table 1. 

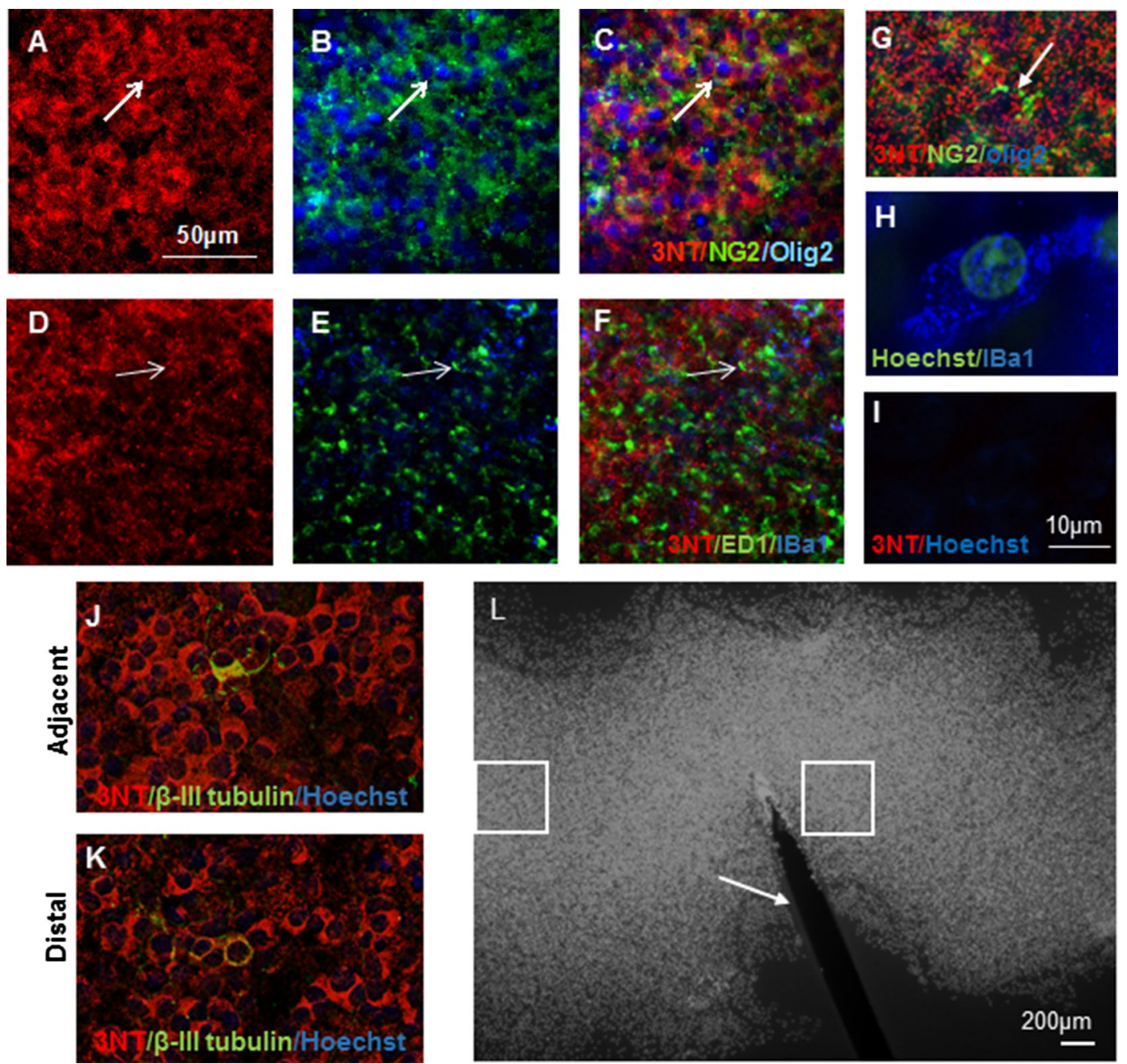

Fig. 2 Representative images of 3NT immunoreactivity $(\mathbf{A}, \mathbf{D})$, colocalised with some NG2/Olig2 + ve cells (B, arrow) with overlay $(\mathbf{C})$, and some ED1/IBA1 - +ve cells $(\mathbf{E}$, arrow) with overlay $(\mathbf{F})$. Higher magnification images of NG2/Olig2 $+(\mathbf{G})$ and IBA+ $(\mathbf{H})$ cells are provided, together with a control image omitting the 3NT primary antibody (I). 3NT immunoreactivity was approximately equivalent both adjacent to the cut injury (J) and more distally (K); $3 \mathrm{NT}$ (red), $\beta$-III tubulin (green), Hoechst nuclear stain (blue). Scale $=50 \mu \mathrm{m}$ for images $\mathbf{A}-\mathbf{F}$, J, $\mathbf{K}$; scale $=10 \mu \mathrm{m}$ for $\mathbf{G}-\mathbf{I}$. A representative image of a spinal cord slice with cut injury, stained with Hoechst nuclear stain (L); boxed areas indicate analysis sites close and distal from the injury (indicated by an arrow), scale $=200 \mu \mathrm{m}$

\section{Discussion}

R/NIR-LT is a prospective antioxidant therapy for preserving function following CNS injury. The treatment is comparably cheap, non-invasive and easy to administer when using LED arrays, and has shown promise in numerous pre-clinical models of CNS injury and for some indications in clinical trials for stroke [20, 24]. However, lack of optimization using comparative studies has probably contributed to the failure of widespread clinical use of R/NIR-LT [24], and there is a need for a standardized, consensus treatment protocol. In this study, we have firstly developed and characterised an in vitro rat model of CNS injury suitable for optimizing treatment strategies for neurotrauma. Secondly we have used the model to compare a range of wavelengths and intensities for efficacy at reducing oxidative stress, in an effort to provide data useful for optimisation of light therapy, with the added advantage of a reduction in animal numbers required. The intensities of light delivered were designed to encompass those shown to be effective in vivo once penetrance of light has been accounted for [39] and were standardised for the number of photons that reached the surface of the cultures for each wavelength utilised. As such, outcomes between wavelengths are directly comparable. Our data suggest $660 \mathrm{~nm}$ light to be the most promising wavelength of those tested for 


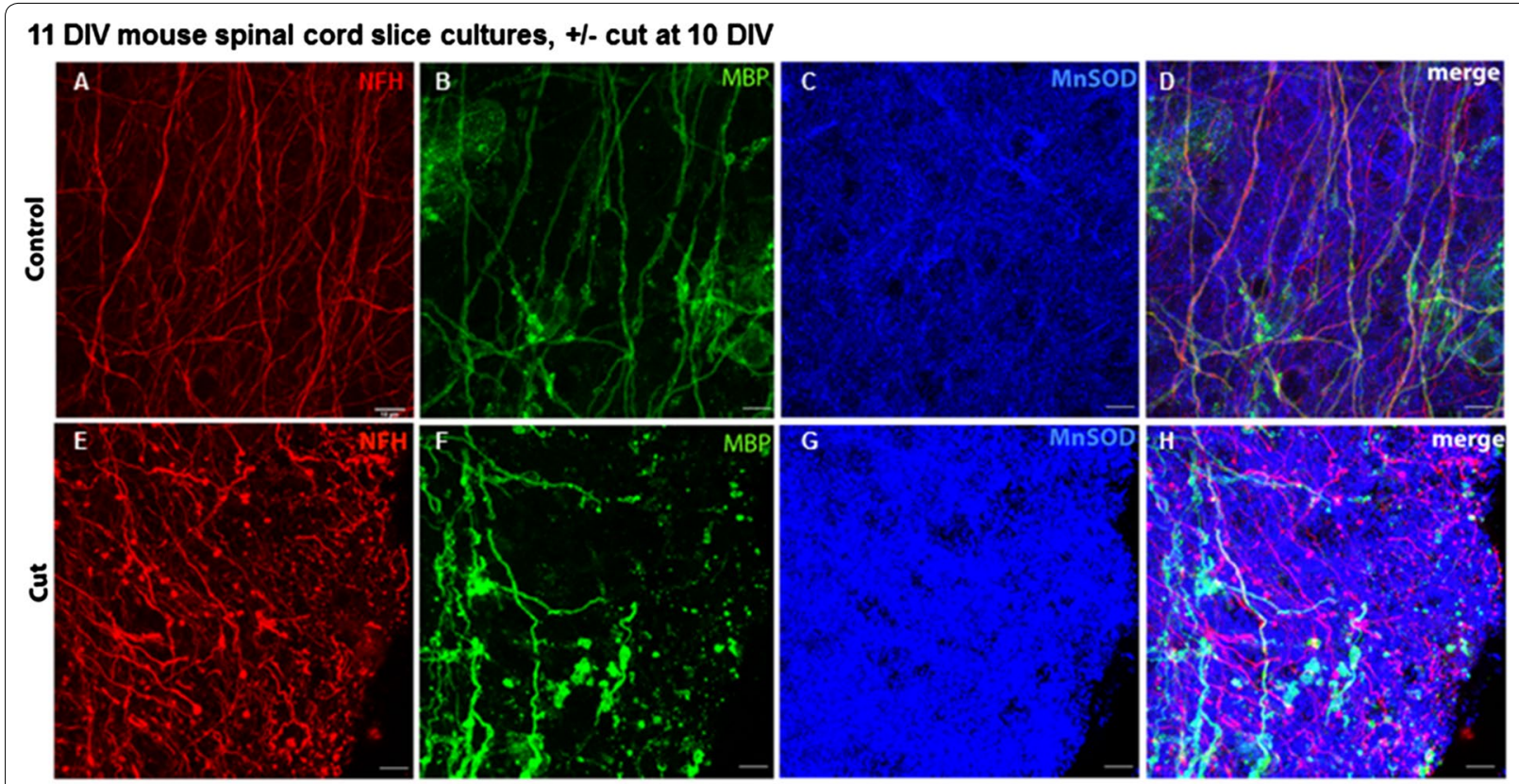

17 DIV mouse spinal cord slice cultures, + cut at 10 DIV
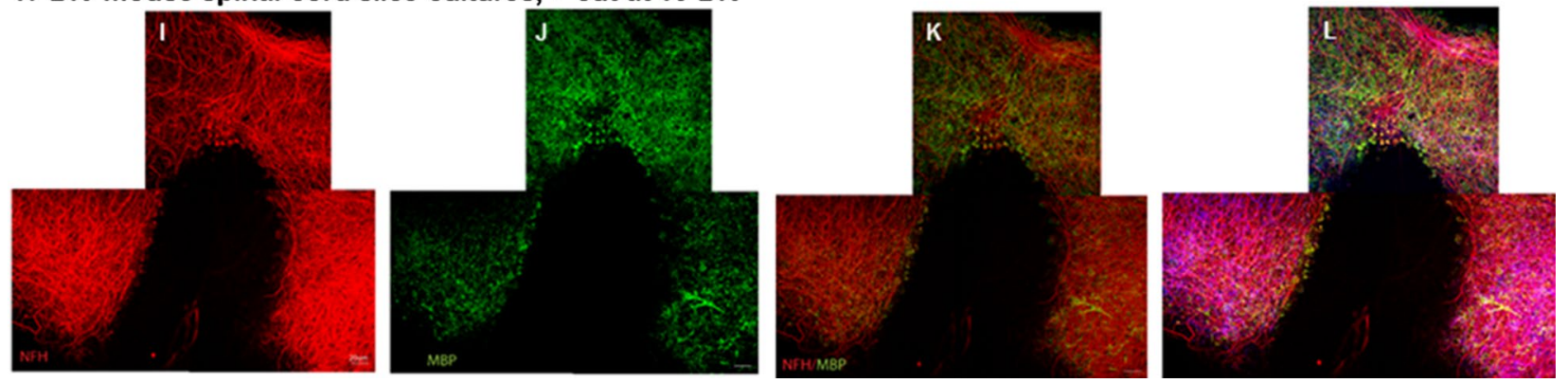

Fig. 3 The effects of cut injury on spinal cord slice cultures from neonatal mouse. Spinal cord slice cultures were cultured for 10 DIV, and either not cut (A-D), or subjected to cut injury (E-H). Immunohistochemical analysis of NFH (red), MBP (green) and MnSOD (blue) with overlay in uncut and cut cultures are shown at 11 DIV $(\mathbf{A}-\mathbf{H})$, scale $=10 \mu \mathrm{m}$. Immunohistochemical analysis of NFH (red), MBP (green) with overlay (I-K) and overlay with Hoechst (L) in cut cultures are shown at 17 DIV $(\mathbf{I}-\mathbf{L})$, scale $=20 \mu \mathrm{m}$

reducing oxidative stress, performing consistently and effectively across all three intensity settings.

When light interacts with biological tissue, photons are either absorbed by cellular constituents or scattered [33], with optimal penetration occurring within an "optical window" [34]. Penetration is dependent on absorption and scattering [33], both of which are greater at shorter, blue/green wavelengths [35]. However, in the current in vitro study, penetrance of the different wavelengths is likely to be relatively uniform and the effects of equivalent numbers of photons of different wavelengths were directly assessed. Different wavelengths of light have been shown to have different effects upon photoacceptors. For example, wavelength bands of 330, 404-420, 680 and $825 \mathrm{~nm}[36,37]$ have been shown to result in oxidation of COX, and distinct wavelength bands of 450, 620 and
$760 \mathrm{~nm}$ reduce COX [36]. Our observation of increased COX activity in vivo following $670 \mathrm{~nm}$ treatment [38], indicate that oxidation of COX by red light increases the activity of the enzyme, at least associated with, and perhaps mediating the therapeutic effect. The results of the current study demonstrating that $660 \mathrm{~nm}$ was the most effective of the tested wavelengths at reducing oxidative stress support these findings and indicate that oxidation of COX may mediate beneficial therapeutic effects.

The other tested wavelengths were outside the optimal wavelengths for COX oxidation, and in the case of $450 \mathrm{~nm}$ were likely to lead to reduction of the enzyme, perhaps accounting for the limited anti-oxidant efficacy. Nevertheless, the highest intensity of light tested in the current study still reduced 3NT immunoreactivity at all four tested wavelengths. Absorption of sufficient energy 


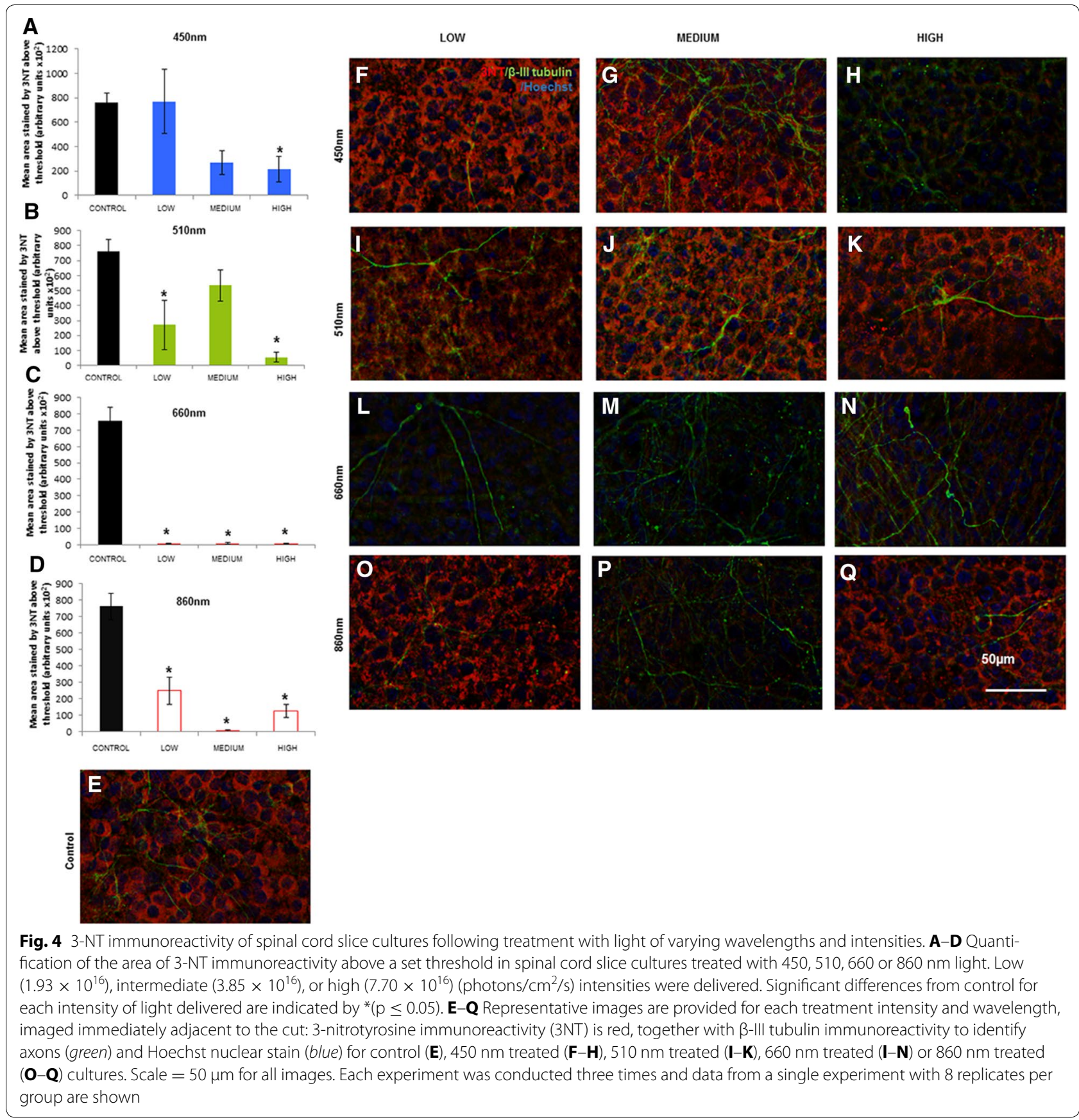

by COX to enable conformational change may have resulted in release of nitric oxide from COX, [14] thereby leading to beneficial effects of wavelengths of light that reduce COX. An alternative mechanism may involve the flavoprotein NADH dehydrogenase (NADH-D), which has been suggested as a photoacceptor involved in blue light therapy [39]. Its action spectra peak at $448 \mathrm{~nm}[40,17]$ corresponds to the blue wavelength setting used in this study $(450 \mathrm{~nm})$. Although activation of
NADH-D $[39,41]$ may promote electron drive through the electron transport chain, this therapeutic effect may be overwhelmed by the detrimental accumulation of superoxide production, perhaps explaining why blue light has produced results reflective of lower efficacy in the current study. It is interesting to note that of the oxidative stress measures assessed, light therapy only resulted in decreases in $3 \mathrm{NT}$ immunoreactivity, indicating that oxidative/nitrosative damage to proteins may be limited 


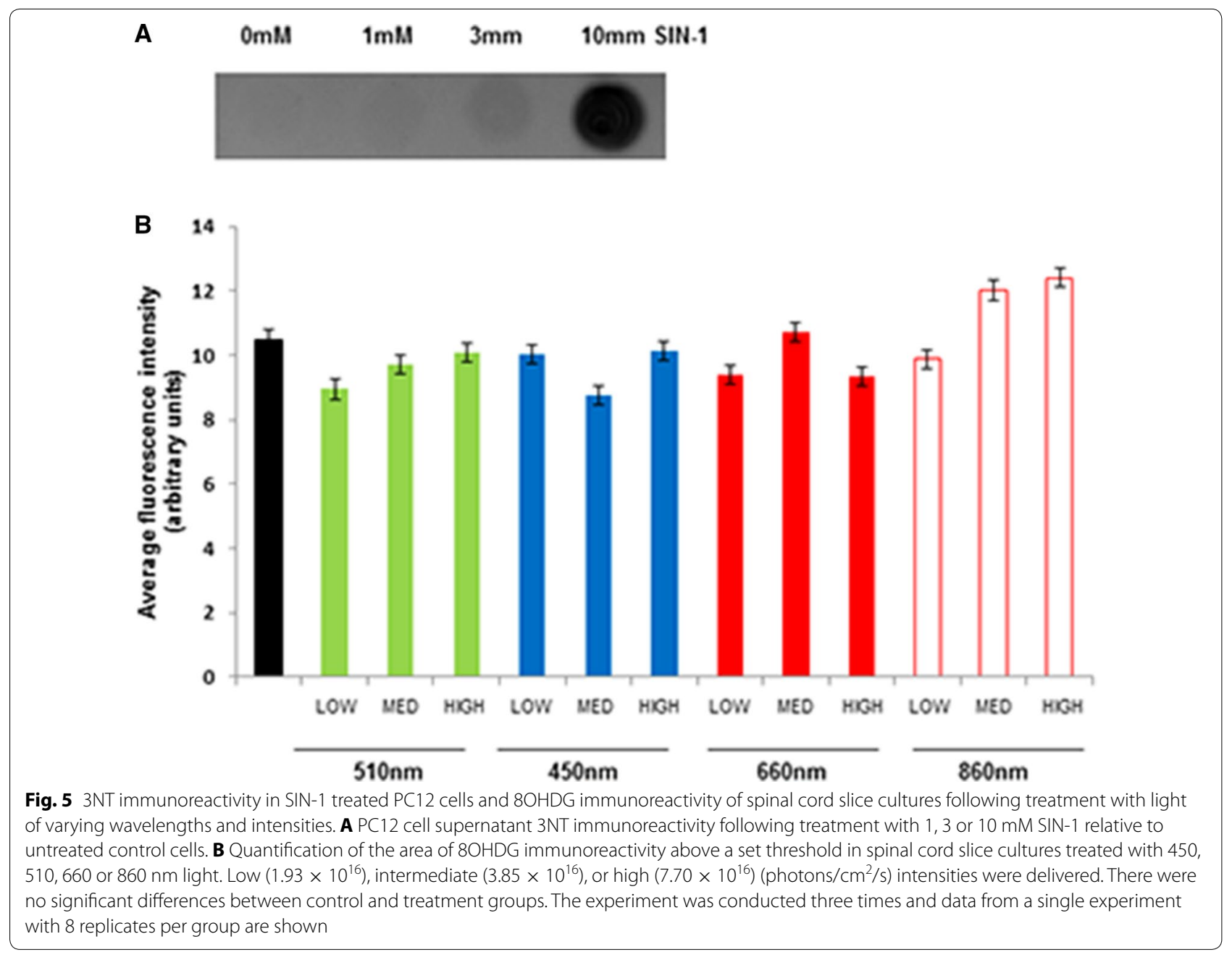

Table 1 Summary of immunohistochemical outcomes

\begin{tabular}{lllll}
\hline & $\begin{array}{l}\text { Increased } \\
\text { with cut }\end{array}$ & $\begin{array}{l}\text { Co-localisation } \\
\text { with MBP }\end{array}$ & $\begin{array}{l}\text { Co-localisation } \\
\text { with microglia/ } \\
\text { macrophages, } \\
\text { OPCs }\end{array}$ & $\begin{array}{l}\text { Effect } \\
\text { of light } \\
\text { treatment }\end{array}$ \\
\hline $8 \mathrm{OHDG}$ & ++ & - & $\mathrm{ND}$ & - \\
$\mathrm{HNE}$ & + & + & $\mathrm{ND}$ & - \\
$3 \mathrm{NT}$ & + & - & + & $\downarrow$ \\
$\mathrm{MnSOD}$ & + & - & $\mathrm{ND}$ & $\mathrm{ND}$
\end{tabular}

+ Is increased immunoreactivity, ++ is strongly increased immunoreactivity, is negative or no effect, ND is not assessed, $\downarrow$ is decreased immunoreactivity

by the mechanisms described above, whereas damage to DNA and lipid oxidation are not. Assessment of antiinflammatory effects of the various wavelengths in the current model would also be interesting to address.

Measures of oxidative damage in the current study rely upon the specificity of the commercial antibodies employed. It is challenging to confirm specific immunostaining of oxidative or nitrosative damage: for example, increased nitrotyrosine immunoreactivity in tissue reflects nitration of tyrosines in a large range of proteins in response to oxidative and nitrosative stress. As such, Western analysis of binding of the antibody will result in a large number of bands and commercial descriptions of these antibodies do not show Western blots. Nevertheless, our observation of increased 3-nitrotyrosine immunoreactivity in the supernatant of cells treated with the peroxynitrate generator SIN-1 [31] indicates that changes in immunoreactivity occur in response to reactive nitrogen species.

Complete transection of the spinal cord, although reflective of complete SCI in patients [42], is rarely seen in the clinic $[43,44]$ and partial transection models are more reflective of clinical SCI. Furthermore, partial transection models are valuable for assessing axonal regeneration as an additional measure in SCI $[45,46]$, and are increasingly used to assess the effects of combinatorial therapies on axonal regeneration post-injury [47]. Our data suggest $660 \mathrm{~nm}$ as an effective wavelength setting 
for light therapy. However in order to ensure delivery of sufficient intensity of light at this wavelength to injured tissue in vivo, it is necessary to assess transmission of the light through the overlying tissue. Calculated and measured assessments of penetrance of irradiation to the spinal cord range from 6 to $11.3 \%$ depending upon wavelength $[11,24,26]$. Together with data from the current study, these values can be used to calculate likely effective dosages of R/NIR-LT to SCI. The lowest effective intensity of $660 \mathrm{~nm}$ light tested here was $1.93 \times 10^{16}$ photons $/ \mathrm{cm}^{2} / \mathrm{s}$. We have measured that the VET75 LED array delivers $5.3 \times 10^{16}$ photons $/ \mathrm{cm}^{2} / \mathrm{s}$ to the surface of the skin when held at $3 \mathrm{~cm}$ above the skin [26]. Given $6.6 \%$ penetrance, we calculate that only approximately $0.35 \times 10^{16}$ photons $/ \mathrm{cm}^{2} / \mathrm{s}$ penetrate to the SCI, perhaps explaining our observed lack of effect of R/NIR-LT following SCI in vivo [26]. The nature of individual CNS injuries may impact upon efficacy of R/NIR-LT, with differing disease pathologies likely differentially modulated by altered photoacceptor activity. For example, increased glial scarring and immune cell infiltration in SCI may overwhelm positive effects of R/NIR-LT on oxidative stress. Nevertheless, it is apparent that transmission of R/NIR-LT must be carefully considered when designing effective R/NIR-LT treatment protocols for CNS injuries.

\section{Conclusions}

Our comparative assessment has resulted in the identification of effective protocols of R/NIR-LT that can be adapted to individual CNS injuries, bearing in mind the transmission properties of the injured and overlying tissue. We have demonstrated that $660 \mathrm{~nm}$ red light is the most effective of the tested wavelengths at reducing oxidative stress, likely due to oxidation of COX. R/NIR-LT at $860 \mathrm{~nm}$ also significantly reduced oxidative stress at all tested intensities. We conclude that although R/NIR-LT appears to be a relatively simple therapy, the choice of dose regime and wavelength utilised needs as much care and attention as designing drug dosing protocols.

\section{Methods}

All procedures involving animals were approved by the University of Western Australia Animal Ethics Committee, Approval number RA3/100/673, or the UK Home Office project licence number $60 / 4524$ and adhered to the National Health and Medical Research Australian Code of Practice for the care and use of animals for scientific purposes. Neonatal PVG rat pups (P0-P5), sex not specified, were procured from the Animal Resources Centre (Murdoch, Western Australia). The mother and pups were housed under standard conditions including $12 \mathrm{~h}$ light/dark cycles and ad libitum access to chow and water.

\section{Spinal cord collection and culture}

Rat or mouse pups at P0-1 days old ( $\mathrm{n}=6$ /experiment) were euthanized using pentobarbital (Virbac, Australia Pty. Ltd., NSW, Australia), administered intraperitoneally at a dose of $800-1000 \mathrm{mg} / \mathrm{kg}(\sim 0.02$ to $0.03 \mathrm{ml})$ as per standard best practice. Cultures were generated from six pups per experiment to ensure sufficient spinal cord slice cultures were available for all experimental groups; cultures were randomly allocated to experimental groups. Each experiment was repeated at least three times. Spinal cords were removed and a $5 \mathrm{~mm}$ segment of cervical-thoracic spinal cord dissected into spinal growth culture media (containing $50 \%$ minimal essential medium, 25 \% heat-inactivated horse serum, 25 \% Earl's balanced salt solution, supplemented with $1 \%$ pen/strep, $1 \%$ glutamax, $0.5 \%$ fungizone, $1.4 \%$ D-glucose). Spinal cords were placed with the posterior surface uppermost and $200 \mu \mathrm{m}$ (for rat) or $300 \mu \mathrm{m}$ (for mouse) longitudinal slices generated using a Mcllwain Tissue Chopper. Three slices were transferred to each Millicell-CM low height cell culture membrane (Millipore, Germany) and cultured in six-well plates; each well containing $1 \mathrm{ml} \mathrm{spi-}$ nal growth culture media. The spinal slices were cultured for 7-17 days at $37{ }^{\circ} \mathrm{C} 5 \% \mathrm{CO}_{2}$, with fresh spinal media supplied every second day. The media change after 6 DIV incorporated $24.8 \mathrm{mM}$ Hepes into the spinal cord media to buffer $\mathrm{pH}$ changes during light therapy. Note that myelinated axons were not observed in slice cultures prepared from P3-4 day old rat pups at 4, 7, 10, 17 or 24DIV (not shown) and cultures from pups of this age were not examined further.

\section{Transection of cultures and R/NIR light treatment}

Phototherapy was administered to the organotypic spinal slices using custom-built LED arrays. The four individual narrow-band LED arrays delivered light with centre wavelengths of 449,516, 662 and $865 \mathrm{~nm}$ (referred to hereafter as 450, 510, 660 and $860 \mathrm{~nm}$, respectively). The emitted light intensity of each device was controlled by adjusting the current supplied to the LEDs and measured using a calibrated CCD spectroradiometer (USB 4000, Ocean Optics) fitted with a cosine collector, allowing calculation of the total number of photons delivered at all emitted wavelengths using the Spectrasuite software program (Ocean Optics). The light output of each LED device was adjusted to produce equal photon irradiance when delivered at a fixed distance of $5 \mathrm{~cm}$ above the spinal slices, with the LED array fixed above the cultures to allow uniform illumination while not touching the culture dish. Light doses were delivered to cultures at three intensities [1.93 × $10^{16}$ (low), $3.85 \times 10^{16}$ (intermediate) and $7.70 \times 10^{16}$ (high) photons $/ \mathrm{cm}^{2} / \mathrm{s}$ ] at each nominal wavelength. Sham-treated control spinal cord slices were 
subject to identical treatment conditions, but the overlying LED device was not switched on. Lower intensities were delivered prior to higher intensities to ensure residual heat was not a confounding factor in low intensity treatments. All cultures were also exposed to normal laboratory lighting conditions during the treatment process; the culture incubator is a dark environment.

After 7 DIV a \#11 scalpel blade was used to make a full depth cut partway through the width of the slice, at the midpoint of each slice, to induce a partial transection insult. Light treatment was applied to the membranes containing the slice cultures for a duration of $30 \mathrm{~min}$, the first treatment delivered immediately following insult and the second $24 \mathrm{~h}$ following insult. Temperature was monitored and maintained at $37{ }^{\circ} \mathrm{C}$ throughout treatment using an incubator, whilst $\mathrm{pH}$ was controlled by the prior addition of Hepes $(24.76 \mathrm{mM})$ to the media. Immediately following the second 30-min light treatment, slices were fixed on the culture membranes for 30 min with $4 \%$ paraformaldehyde in $0.1 \mathrm{M}$ phosphate buffer and washed with phosphate buffered saline (PBS). Slices on membrane were cut out and stored at $4{ }^{\circ} \mathrm{C}$ in a 24 well plate containing PBS azide ( $0.1 \%$ sodium azide in sterile PBS, $1 \mathrm{ml} /$ well) for future immunohistochemical analyses.

\section{Immunohistochemical analyses}

Immunohistochemical analyses were conducted on spinal slices still adhered to the membranes according to established procedures [48], using primary antibodies recognizing: 3NT(1:500; Abcam, Cambridge, UK); $\beta$-III Tubulin (1:500; Covance); neurofilament (NFH) (1:50,000; Encor); 8OHDG (1:500; Abcam, Cambridge, UK); HNE (1:200; Jomar Bioscience); MnSOD (1:500, StressGen); MBP (1:500, Abcam); Olig2 (1:500; Abcam); NG2 (1:250; Invitrogen); IBA1 (1:500; Novachem), ED-1 (1:500; Millipore) incubated overnight with the slices at $4{ }^{\circ} \mathrm{C}$. Secondary antibodies were species-specific AlexaFluor $^{\circledR}$ 555- and 488-conjugated (1:400; Invitrogen), with the addition of Hoechst nuclear stain (1:1000; Invitrogen, VIC); incubated for $2 \mathrm{~h}$ at room temperature.

The specificity of the $3 \mathrm{NT}$ antibody was assessed using PC12 cells grown in RPMI medium supplemented with $10 \%$ horse serum, $5 \%$ fetal bovine serum, $2 \mathrm{mM} \mathrm{L-glu-}$ tamine, $2 \mathrm{mM}$ penicillin-streptomycin, $1 \mathrm{mM}$ MEM sodium pyruvate and $0.1 \mathrm{mM}$ MEM non-essential amino acids (GIBCO ${ }^{\circledR}$, Invitrogen, Carlsbad, California, USA), on flasks coated with poly-L-lysine (PLL; $10 \mu \mathrm{g} / \mathrm{ml}$; Sigma, St Louis, Missouri, USA) and housed in an incubator at $37{ }^{\circ} \mathrm{C}, 5 \% \mathrm{CO}_{2}$. Cells were seeded at $4 \times 10^{4}$ cells/well of a PLL coated 24 well plate, allowed to adhere and then incubated with 1, 3 or $10 \mathrm{mM} \mathrm{SIN-1} \mathrm{(Sigma-}$ Aldrich) for 24 h. $5 \mu \mathrm{l}$ samples of resultant cell culture supernatants were assessed by dot-blot.

\section{Microscopy and image analysis}

For each experiment, intensity of immunoreactivity was visualized and imaged in a single session using a Nikon Eclipse Ti Inverted Microscope (Nikon Corporation, Japan) or a Leica SP7 confocal: images were captured at constant exposure settings across all treatment groups. Each experiment was repeated at least three times, using cultures prepared on different days. Images were captured at areas both close and distal to the injury site, with two close and two distal images taken per slice. Two spinal cord slices were imaged per treatment group, generating eight data points for each group. Each image consisted of a stack of 13 visual slices captured at $0.5 \mu \mathrm{m}$ increments along the $\mathrm{z}$-axis, and obtained from the middle $6 \mu \mathrm{m}$ of the slice. Images captured using the nonconfocal microscope were deconvoluted using autoquant blind deconvolution with Nikon Elements AR software. Image analysis was conducted on a single central visual slice using Image//Fiji analysis software, setting constant arbitrary threshold intensities for all images in an analysis and determining the area above the threshold intensity and the mean intensity of fluorescence of the image.

\section{Statistical analysis}

All data are displayed as mean \pm standard error of the mean (SEM). Statistical analyses were conducted using SPSS statistical software. Data from regions both close and distal to the transection were combined, as statistical analysis using nonparametric Wilcoxon pairedsamples test revealed no significant differences between the regions ( $\mathrm{p} \leq 0.05)$. Equality of variance F-tests were conducted to test for homogeneity of variance in groups within experiments. Collated data were analysed by comparing treated to control using analysis of variance (ANOVA) with Games Howell post hoc test at $95 \%$ confidence intervals $(\mathrm{p} \leq 0.05)$. Statistical assessments were conducted independently for each experiment and representative analyses shown; it is not possible to average immunohistochemical intensity data from different experiments due to inter-experiment variability in baseline values.

\section{Abbreviations \\ ANOVA: analysis of variance; CNS: central nervous system; COX: cytochrome $c$ oxidase; DIV: days in vitro; HNE: hydroxynonenal; 8OHDG: 8-hydroxyguanosine; LED: light emitting diode; MBP: myelin basic protein; MnSOD: manganese superoxide dismutase; NADH-D: NADH dehydrogenase; NEST: NeuroThera Effectiveness and Safety Trial; NFH: neurofilament; 3NT: 3-nitrotyrosine; P: postnatal day; PBS: phosphate buffered saline; PVG: Piebald Virol Glaxo; RNS: reactive nitrogen species; ROS: reactive oxygen species; R/NIR-LT: red/near- infrared light therapy; SCI: spinal cord injury; SEM: standard error of the mean; TBI: traumatic brain injury.}

\section{Authors' contributions}

BEA conducted experiments, analysed data, contributed to writing manuscript. ES, CAB, MKG, SS conducted experiments and analysed data. NSH, AW, 
MF designed and conducted experiments, analysed data and contributed to writing of manuscript. All authors read and approved the final manuscript.

\section{Author details}

${ }^{1}$ Experimental and Regenerative Neurosciences, School of Animal Biology, The University of Western Australia, Crawley, WA, Australia. ${ }^{2}$ Department of Biology and Biochemistry, The University of Bath, Bath, UK. ${ }^{3}$ Centre for Regenerative Medicine, University of Edinburgh, Edinburgh, UK. ${ }^{4}$ Department of Biological Sciences, Macquarie University, Sydney, NSW 2109, Australia.

\section{Acknowledgements}

This project is funded through the Road Trauma Trust Account, Western Australia, but does not reflect views or recommendations of the Road Safety Council.

\section{Competing interests}

The authors declare that they have no competing interests.

\section{Availability of data and materials}

All the data supporting our findings is contained within the manuscript.

\section{Ethics and consent to participate}

All procedures involving animals were approved by the University of Western Australia Animal Ethics Committee, Approval number RA3/100/673, or the UK Home Office project licence number 60/4524 and adhered to the National Health and Medical Research Australian Code of Practice for the care and use of animals for scientific purposes.

\section{Funding}

We acknowledge financial support from the Neurotrauma Research Program of Western Australia, an initiative of the Road Safety Council of Western Australia. MF is supported by an NHMRC Career Development Fellowship (APP1087114). The funding bodies played no part in the design of the study and collection, analysis, and interpretation of data or in writing the manuscript.

Received: 6 September 2015 Accepted: 11 May 2016

Published online: 18 May 2016

\section{References}

1. Park E, Velumian AA, Fehlings MG. The role of excitotoxicity in secondary mechanisms of spinal cord injury: a review with an emphasis on the implications for white matter degeneration. J Neurotrauma. 2004;21:754-74

2. Carrico KM, Vaishnav R, Hall ED. Temporal and spatial dynamics of peroxynitrite-induced oxidative damage after spinal cord contusion injury. J Neurotrauma. 2009;26:1369-78.

3. Doig RLH, Bartlett CA, Maghzal GJ, Lam M, Archer M, et al. Reactive species and oxidative stress in optic nerve vulnerable to secondary degeneration. Exp Neurol. 2014;261:136-46.

4. Kowaltowski AJ, de Souza-Pinto NC, Castilho RF, Vercesi AE. Mitochondria and reactive oxygen species. Free Radic Biol Med. 2009;47:333-43.

5. Xiong Y, Hall ED. Pharmacological evidence for a role of peroxynitrite in the pathophysiology of spinal cord injury. Exp Neurol. 2009;216:105-14.

6. Lewen A, Matz P, Chan PH. Free radical pathways in CNS injury. J Neurotrauma. 2000;17:871-90.

7. Cuzzocrea S, Genovese T. Role of free radicals and poly (ADP-ribose) polymerase-1 in the development of spinal cord injury: new potential therapeutic targets. Curr Med Chem. 2008;15:477-87.

8. Gandhi S, Abramov AY. Mechanism of oxidative stress in neurodegeneration. Oxid Med Cell Longev. 2012;2012:428010.

9. Barrera G. Oxidative stress and lipid peroxidation products in cancer progression and therapy. ISRN Oncol. 2012;2012:137289.

10. Salgado S, Parreira R, Ceci L, de Oliveira L, Zangaro R. Transcranial light emitting diode therapy (TCLT) and its effects on neurological disorders. J Bioeng Biomed Sci. 2015;5:2.
11. Byrnes KR, Waynant RW, Ilev IK, Wu X, Barna L, et al. Light promotes regeneration and functional recovery and alters the immune response after spinal cord injury. Lasers Surg Med. 2005;36:171-85.

12. Natoli R, Zhu Y, Valter K, Bisti S, Eells J, et al. Gene and noncoding RNA regulation underlying photoreceptor protection: microarray study of dietary antioxidant saffron and photobiomodulation in rat retina. Mol Vis. 1801;2010:16

13. Albarracin RS, Valter K. Treatment with 670-nm light protects the cone photoreceptors from white light-induced degeneration. In: LaVail MM, Ash JD, Anderson RE, Hollyfield JG, Grimm C, editors. Retinal degenerative diseases. Berlin: Springer; 2012. p. 121-8.

14. Hashmi J, Huang Y, Osmani B, Sharma S, Naeser M, et al. Role of low-level laser therapy in neurorehabilitation. PM\&R. 2010;2:S292-305.

15. Wu X, Dmitriev AE, Cardoso MJ, Viers-Costello AG, Borke RC, et al. $810 \mathrm{~nm}$ Wavelength light: an effective therapy for transected or contused rat spinal cord. Lasers Surg Med. 2009;41:36-41.

16. Hamblin MR, Demidova TN. Mechanisms of low level light therapy. In: International society for optics and photonics. p. 614001-12.

17. Karu T. Molecular mechanism of the therapeutic effect of low-intensity laser radiation. Lasers Life Sci. 1988;2:53-74.

18. Karu T. Laser biostimulation: a photobiological phenomenon. J Photochem Photobiol B. 1989;3:638-40.

19. Karu T, Pyatibrat L, Kalendo G. Irradiation with He-Ne laser increases ATP level in cells cultivated in vitro. J Photochem Photobiol B. 1995;27:219-23.

20. Szymanski CR, Chiha W, Morellini N, Cummins N, Bartlett CA, et al. Paranode abnormalities and oxidative stress in optic nerve vulnerable to secondary degeneration: modulation by $670 \mathrm{~nm}$ light treatment. PLoS One. 2013;8:e66448.

21. Mester $E$, Jaszsagi NE. The effect of laser radiation on wound healing and collagen synthesis. Studia Biophys. 1973;35:227-30.

22. Chung H, Dai T, Sharma SK, Huang Y-Y, Carroll JD, et al. The nuts and bolts of low-level laser (light) therapy. Ann Biomed Eng. 2012;40:516-33.

23. Hacke W, Schelinger P, Albers G, Bornstein N, Dahlof B, et al. Transcranial laser therapy (TLT) for acute Ischemic stroke. Results of NEST 3, a pivotal phase III randomized clinical trial (RCT). Karger Allschwilerstrasse 10, CH-4009 Basel, Switzerland; 2013. p. 191.

24. Fitzgerald M, Hodgetts S, Van Den Heuvel C, Natoli R, Hart NS, et al. Red/ near-infrared irradiation therapy for treatment of central nervous system injuries and disorders. Rev Neurosci. 2013;24:205-26.

25. Jenkins PA, Carroll JD. How to report low-level laser therapy (LLLT)/photomedicine dose and beam parameters in clinical and laboratory studies. Photomed Laser Surg. 2011;29:785-7.

26. Giacci MK, Wheeler L, Lovett S, Dishington E, Majda B, et al. Differential effects of 670 and $830 \mathrm{~nm}$ red near Infrared Irradiation therapy: a comparative study of optic nerve injury, retinal degeneration, traumatic brain and spinal cord injury. PLoS One. 2014;9:e104565.

27. Lee Y, Morrison BM, Li Y, Lengacher S, Farah MH, et al. Oligodendroglia metabolically support axons and contribute to neurodegeneration. Nature. 2012;487:443-8.

28. Zhang $H$, Jarjour AA, Boyd A, Williams A. Central nervous system remyelination in culture-a tool for multiple sclerosis research. Exp Neurol. 2011;230:138-48

29. Giacci MK, Hart NS, Hartz RV, Harvey AR, Hodgetts SI, et al. Method for the assessment of effects of a range of wavelengths and intensities of red/near-infrared light therapy on oxidative stress in vitro. J. Vis. Exp. 2015;97:e52221.

30. Hashmi JT, Huang Y-Y, Sharma SK, Kurup DB, De Taboada L, et al. Effect of pulsing in low-level light therapy. Lasers Surg Med. 2010;42:450.

31. Trackey JL, Uliasz TF, Hewett SJ. SIN-1-induced cytotoxicity in mixed cortical cell culture: peroxynitrite-dependent and -independent induction of excitotoxic cell death. J Neurochem. 2001;79:445-55.

32. Yasuda S, Idell S, Liu MC. Generation and release of nitrotyrosine O-sulfate by HepG 2 human hepatoma cells upon SIN-1 stimulation: identification of SULT1A3 as the enzyme responsible. Biochem J. 2007;401:497-503.

33. Cheong W-F, Prahl SA, Welch AJ. A review of the optical properties of biological tissues. IEEE J Quantum Electron. 1990;26:2166-85.

34. Parrish JA. New concepts in therapeutic photomedicine; photochemistry, optical targeting and the therapeutic window. J Investig Dermatol. 1981;77:45-50. 
35. Arora P, Sarkar R, Garg VK, Arya L. Lasers for treatment of melasma and post-inflammatory hyperpigmentation. J Cutan Aesthet Surg. 2012;5:93.

36. Karu T. Primary and secondary mechanisms of action of visible to near-IR radiation on cells. J Photochem Photobiol B. 1999;49:1-17.

37. Szundi I, Liao G-L, Einarsdóttir Ó. Near-infrared time-resolved optical absorption studies of the reaction of fully reduced cytochrome c oxidase with dioxygen. Biochemistry. 2001;40:2332-9.

38. Szymanski CR, Chiha W, Morellini N, Cummins N, Bartlett CA, et al. Paranode abnormalities and oxidative stress in optic nerve vulnerable to secondary degeneration: modulation by $670 \mathrm{~nm}$ light treatment. PLoS One. 2013:8:e66448.

39. Gao X, Xing D. Molecular mechanisms of cell proliferation induced by low power laser irradiation. J Biomed Sci. 2009;16:1-16.

40. Wakao H, Wakagi T, Oshima T. Purification and properties of NADH dehydrogenase from a thermoacidophilic archaebacterium, Sulfolobus acidocaldarius. J Biochem. 1987;102:255-62.

41. Vani T, Raghavendra A. Tetrazolium reduction by guard cells in abaxial epidermis of Vicia faba: blue light stimulation of a plasmalemma redox system. Plant Physiol. 1989;90:59-62

42. Nakae A, Nakai K, Yano K, Hosokawa K, Shibata M, et al. The animal model of spinal cord injury as an experimental pain model. BioMed Res Int. 2011;2011:939023.
43. Talac R, Friedman J, Moore M, Lu L, Jabbari E, et al. Animal models of spinal cord injury for evaluation of tissue engineering treatment strategies. Biomaterials. 2004:25:1505-10.

44. Poon PC, Gupta D, Shoichet MS, Tator CH. Clip compression model is useful for thoracic spinal cord injuries: histologic and functional correlates. Spine. 2007;32:2853-9.

45. Kwon BK, Oxland TR, TetzlaffW. Animal models used in spinal cord regeneration research. Spine. 2002;27:1504-10.

46. Sharif-Alhoseini M, Rahimi-Movaghar V. Animal models in traumatic spinal cord injury. In: Dionyssiotis Y, editor. Topics in Paraplegia. InTech; 2014. doi:10.5772/57189. Available from: http://www.intechopen.com/books/ topics-in-paraplegia/animal-models-in-traumatic-spinalcord-injury.

47. Kundi S, Bicknell R, Ahmed Z. Spinal cord injury: current mammalian models. Am J Neurosci. 2013:4:1-12.

48. Fitzgerald M, Bartlett CA, Harvey AR, Dunlop SA. Early events of secondary degeneration after partial optic nerve transection: an immunohistochemical study. J Neurotrauma. 2010;27:439-52.

\section{Submit your next manuscript to BioMed Central and we will help you at every step:}

- We accept pre-submission inquiries

- Our selector tool helps you to find the most relevant journal

- We provide round the clock customer support

- Convenient online submission

- Thorough peer review

- Inclusion in PubMed and all major indexing services

- Maximum visibility for your research

Submit your manuscript at www.biomedcentral com/submit 